1708 INCIDENCE OF INTRAVENTRICULAR HEMORRHAGE BY 100 Losure, Chari Otis, Soraya Abbasi and Ronald Librizzi Neonatology, Univ. of Pa. Sch. of Med., Dept. of Ped. and Ob/Gyn, Pennsylvania Hosp., Phila., Pa. The optimal method for delivery of the infant less than $1500 \mathrm{grams}$ is still under debate. dn order to assess this situation we examined the records of all In order to assess this situation we examined the records of all month period, 226 infants were delivered between 500 and 1500 gms. A total of 91 infants were born vaginally and 135 by $\mathrm{C} /$ section. All infants were inborn. The overall survival rate was $85 \%$. For this study only non-asphyxiated infants ( 5 minute Apgar $\geq 7$ ) were included, for a total of 155 infants. There were 60 infants born vaginally and 95 born by $\mathrm{C} /$ section. The mean birth weights were $1161 \pm 255 \mathrm{gms}$ and $1153 \pm 231 \mathrm{gms}$ respectively. All infants had a routine cranial ultrasound during the first 72 hours of life, and then weekly thereafter as indicated. The overall incidence of intraventricular hemorrhage (IVH) was $12 \%$. The results by delivery were as follows:

\begin{tabular}{cccc} 
& $\operatorname{VAG}(\mathrm{N}=60)$ & $\mathrm{C} / \mathrm{S}(\mathrm{N}=95)$ & \\
\hline IVH & $11(18 \%)$ & $6(6 \%)$ & n.s. \\
\hline Grade I-II & 8 & 5 & \\
Grade III-IV & 3 & 1 &
\end{tabular}

Gre The incldence of IVH, diagnosed after the fifth day of life was $8 \%$ in both groups. This would indicate neonatal rather numbers ultimately were small and there was no difference in survival noted, delivery tended to favor $\mathrm{C} /$ section.
1711 ISOLATED VENTRICLES AFTER IVH. J.F. Pasternak and I.W. Eller. (spo

Post hemorrhagic hydrocephalus (PHH) frequently occurs after large neonatal IVH. Previous experience has documented the difficulty in controlling progressive ventricular dilatation soon after the hemorrhage, necessitating trials of multiple medical (glycerol, diamox, LP, etc) and surgical (extraventricular drainage, ventricular reservoir, etc) therapies. Our experience indicates that PHH continues to pose difficulties in management even after a definitive ventriculoperitoneal shunt (VPS) has been accormplished. Five babies required VPS for PHH during a 12 month period, 3 after grade III and 2 after grade 4 IVH. All babies were treated with serial LP and 3 were treated with a ventricular reservoir prior to VPS. During the first 6 months after VPS, 3 babies required the insertion of additional VPS for isolated ventricles. One infant required bilateral lateral ventricle VPS because of isolation of a lateral ventricle, one required insertion of a posterior fossa shunt to treat an isolated 4th ventricle, and one infant required bilateral lateral ventricle shunts and a posterior fossa shunt because of isolation of the 4th ventricle and a temporal hom. Despite progressive dilatation of the isolated ventricle, neither accelerated head growth nor signs of increased intracranial pressure were seen. Symptoms were subtle initially, consisting only of vague irritability and inattention. The frequency of isolated ventricles in PHH demands that these infants be carefully watched even after VPS. The paucity of signs and symptoms requires frequent CT or ultrasound even if the clinical condition seems stable.
1709 NUCLEAR MAGNETIC RESONANCE IN INTRACRANIAL DISEASE OF CHILDHOOD. Authors: Roger J. Packer, Robert A. Zimmer-
man, Larissa T. Bilanuik. Children's Hospital of Philadelphia, Divisions of Neurology and Neuroradiology, Phila. Nuclear magnetic resonance (NMR) is a non-invasive technique which avoids the hazards of x-irradiation and the need for intravenous contrast material. It seems an ideal imaging tool for the pediatric population. We studied 60 patients with central nervous system disease, median 8 years (range 6 days to 21 years) on a proton 0.125 Telsa NMR machine. Images were obtained in the transverse, horizontal and coronal planes by the partial saturation technique ( $\mathrm{T}_{1}$ and $\mathrm{T}_{2}$ welghed images done in selected cases). NMR results were compared to concurrent enhanced computed tomographic (eCT) studies and other radiographic techniques in all cases. Patients included those with primary brain neoplasms (45), spinal cord tumors (4), intracerebral hemorrhage (3), Chiari malformation (2), demyelinating disease (2), and congenital brain anomalies (4). We conclude that: 1) NMR is a sensitive means of anomalies (4). We conclude that: 1) NMR is a sensitive means of visualizing structural CNS disease; 2) NMR often supplies anatomic information not demonstrable by CT or other radiograpbic cord; 3) NMR is well tolerated by pediatric patients; and 4) NMR although demonstrating abnormality as frequently as eCT, of ten is less specific for the type of pathology present. NMR, because of its safety and sensitivity, may soon be the imaging procedure of choice in the initial evaluation of children with CNS disease (especially in the posterior fossa and upper cervical cord), but will not completely replace the need for other imaging techniques.

\section{+1710 HEMORRHAGIC AND ISCHEMIC LESIONS IN THE PRETERM}

BRAIN, Yehoshua Zarfin, Karen Pape, Chukwuma Nwaesei, David Martin, Alan Daneman, Wendy Murphy.

(Spon. by P.M. Fitzhardinge). Research Institute, Hosp. for Sick children, Depts. Pediatrics and Radiology, University of Toronto. During 1 year, 77 infants of gestation $<37$ wks survived severe asphyxia, primary apnea or seizures. Sequential brain ultrasound scans were done to determine the incidence and evolution of hemorrhage in the germinal layer (GLH), ventricles (IVH) or cerebral white matter (ICH) and ischemic periventricular leukomalacia (PVL).

\begin{tabular}{|c|c|c|c|}
\hline & Group 1 & Group 2 & Group 3 \\
\hline Gest. age (wk) & $25-28$ & $29-32$ & $33-36$ \\
\hline . of Patients & 26 & 26 & 25 \\
\hline GLH (Gr. I ) & $1246 \%$ & $1662 \%$ & $936 \%$ \\
\hline IVH $(G r . I I, I I I)$ & $1558 \%$ & $935 \%$ & $28 \%$ \\
\hline ICH (Gr. IV) & $831 \%$ & $8 \frac{\circ}{5}$ & -- \\
\hline PVL & $1558 \%$ & $935 \%$ & $1144 \%$ \\
\hline
\end{tabular}

Groups 1 and 2 (vs 3) had an increased incidence of GLH ( $p<0.05$ ) and IVH (p $(0.01)$. ICH and major IVH occurred most frequently in Group 1 (vs $2 \& 3$, p $<0.05$ ). PVL diagnosed by white matter echogenicity or cysts occurred with equal frequency across all gestational ages. All cases of IVH and ICH and all but 2 cases of GLH were diagnosed within 14 days of age. PVL superolateral to the ventricle developed later (up to age 67 days) and would have been missed by early scanning. The data suggest that all high risk cases should have additional scans 3 to 6 wks after the insult.
ERYTHROCYTE CHEMILUMINESCENCE DEFECT IN DUCHENNE

1712 MUSCULAR DYSTROPHY AND CHRONIC GRANULOMATOUS DISEASE. Allen G. Peerless, E. Richard Stiehm, University of Callfornia-Los Angeles, Callfornia, Department of Pediatrics.

We previously demonstrated abnormal opsonized zymosan (OZ) and concanavalin A (con-A) stimulated chemiluminescence (CL) in purified suspensions of neutrophils (PMN), PMN membranes, erythrocytes (RBC), RBC membranes, and T-lymphocytes from patients with chronic granulomatous disease (CGD), suggesting generalization of the PMN membrane defect to other cell types (Ped. Res. 17: $1022,1983)$. Duchenne muscular dystrophy (DMD) resembles CGD in its X-1inked transmission, carrier mosaicism, membrane localization of the metabolic defect, and similarity to the rare McLeod phenotype, a state of weakened antigenicity of Kell RBC antigens and absence of leukocyte and RBC Kx antigen. We therefore studied the CL responses of intact and membrane preparatiors from $\mathrm{PMN}$ and $\mathrm{RBC}$, to $\mathrm{OZ}$ and con-A stimulation, in 10 males with DMD. Results are expressed as peak stimulated response in counts per minute $t$ standard error of the mean $\times 10^{-3}$.

\begin{tabular}{|c|c|c|c|c|c|c|}
\hline Fraction & $\overline{P M N}$ & PMN mem & $\mathrm{RBC}$ & $\mathrm{RBC}$ mem & $\mathrm{RBC}$ & RBC mem \\
\hline Stimulus & $\mathrm{OZ}$ & $\mathrm{OZ}$ & $\mathrm{OZ}$ & $\mathrm{OZ}$ & $\mathrm{C}-\mathrm{A}$ & $\mathrm{C}-\mathrm{A}$ \\
\hline Luminol & - & + & + & + & + & + \\
\hline 7 & 225 & $1898+491$ & $155+54$ & $39+1$ & $40+1$ & $37+1$ \\
\hline ormal & $183 \mp 85$ & $816 \pm+\overline{6} 06$ & $122+47$ & $39+4$ & $23 \overline{0}+159$ & $53 \overline{ \pm 5}$ \\
\hline$P$ value & $<0.08$ & $<0.03$ & $<0 . \overline{8}$ & $<0.9$ & $<0.1$ & $<0,001 *$ \\
\hline
\end{tabular}

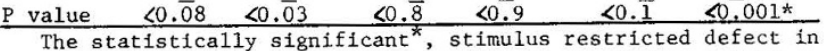
$\mathrm{RBC}$ membrane CL is consistent with other reported abnormalities in the RBC of DMD subjects and may relate to disruption of membrane respiratory metabolism, analagous to CGD.

1713 (IVH) ON HEAD GROWTH VELOCITY OF PREMATURE INFANTS. G.R. Pereira, J. Hoffman, J. Bernbaum, $M$. Georgieff, M. Hoffman-Williamson, A. Daft. (Spon. by W.W.Fox). Dept. o Peds., Children's Hosp, of Phila. and Univ. of Pa. Sch. of Med., Phila., PA. Head growth velocity was assessed weekly during the neonatal period and at 3 month intervals up to one year of corrected age (CA) in 47 premature infants whose clinical data is shown below (mean + SEM):

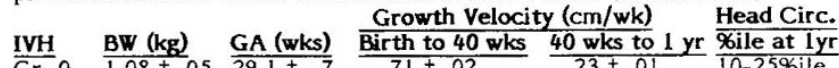
Gr.0 $1.08 \pm .05 \quad 29.1 \pm .7 \quad \frac{.71 \pm .02}{.23 \pm .01} \frac{10-25 \% \text { ile }}{10}$ Gr. $1 \quad 1.38 \pm .05 \quad 30.4 \pm .6 \quad .69 \pm .07 \quad .21 \pm .02 \quad 10-25 \%$ ile Gr. $2 \quad 1.07 \pm .9 \quad 29.2 \pm .7 \quad .64 \pm .02 \quad .24 \pm .02 \quad$ l0\%ile $.67 \pm .02 \quad .25 \pm .01 \quad 5-10 \%$ il

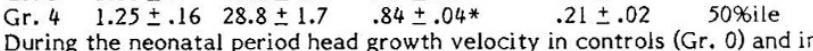
infants with grade 1,2, and 3 IVH were comparable but below intrauterine standards $(0.76 \mathrm{~cm} /$ week). Infants with grade $4 \mathrm{IVH}$ had significantly higher head growth rates than all other groups $(0.84 \mathrm{~cm} /$ week, $\mathrm{p}<0.05)$. The neonatal pattern of head growth in infants with grade 4 IVH was not uniform: 2 of these infants (shunted) exhibited head growth above the $90 \%$ ile, while 1 infant (shunted) had head growth below the $3 \%$ ile and 3 infants (1 shunted) demonstrated head growth within normal percentiles. At follow-up the same patterns in head growth established during the neonatal period persisted up to year of CA. However, at that time, the head growth velocity in infants with grade $4 \mathrm{IVH}$ was no longer significantly different. This study demonstrates that in the absence of grade 4 IVH there is not detectable adverse effect of IVH on head growth velocity of premature infants up to one year of corrected age. 